\title{
$\dot{\nabla}_{\text {Nursingal Academic }}^{\text {Glournal }}$
}

\section{Boas práticas de enfermagem na utilização de dreno de tórax: revisão integrativa}

\author{
Good practices in nursing in the use of chest tubes: an integrative review
}

Buenas prácticas de enfermería en el uso de sondas torácicas: una revisión integradora

\author{
Barbara Nino Ornellas \\ Hasselmann ${ }^{1}$ \\ ORCID: 0000-0002-6925-6704 \\ Cyntia da Silva Ranção ${ }^{1}$ \\ ORCID: 0000-0003-1087-0504 \\ Graziela Silva Tavares ${ }^{1}$ \\ ORCID: 0000-0003-0328-6086 \\ Luana Ferreira de Almeida ${ }^{1}$ \\ ORCID: 0000-0001-8433-4160 \\ Flávia Giron Camerini ${ }^{1}$ \\ ORCID: 0000-0002-4330-953X \\ Vanessa Galdino de Paula ${ }^{1}$ \\ ORCID: 0000-0002-7147-5981 \\ ${ }^{1}$ Universidade do Estado do Rio \\ de Janeiro. Rio de Janeiro, Brasil.
}

Como citar este artigo:

Hasselmann BNO, Ranção CS, Tavares

GS, Almeida LF, Camerini FG, Paula

VG. Boas práticas de enfermagem na utilização de dreno de tórax: revisão integrativa. Glob Acad Nurs.

2021;2(Sup.2):e173.

https://dx.doi.org/10.5935/2675-

5602.20200173

Autor correspondente:

Barbara Nino Ornellas Hasselmann

E-mail:

barbara_onellas@yahoo.com.br

Editor Chefe: Caroliny dos Santos Guimarães da Fonseca

Editor Executivo: Kátia dos Santos Armada de Oliveira

Submissão: 01-05-2021

Aprovação: 24-05-2021

\section{Resumo}

Objetivou-se identificar na literatura científica as melhores práticas de enfermagem relacionadas a utilização de dreno torácico em Unidade de Terapia Intensiva adulta. Trata-se de uma revisão integrativa com consulta à Literatura Latino-Americana do Caribe em Ciências da Saúde, Base de Dados de Enfermagem, Sistema Online de Busca e Análise de Literatura Médica, Scientific Electronic Library Online, PubMed, CINAHL e EBSCO. Incluídos estudos publicados entre janeiro de 2014 a dezembro de 2018 nos idiomas inglês, português e espanhol e disponíveis na íntegra com textos completos. Excluídos aqueles com pacientes pediátricos e duplicidades. Foram identificados 133 estudos que deram origem a cinco artigos analisados. Dos artigos selecionados $80 \%$ corresponderam a estudos internacionais; $40 \%$ publicados em 2016 e $60 \%$ com análise quantitativa. Emergiram as categorias de boas práticas com dreno torácico, complicações relacionadas ao dreno torácico e uso de protocolo de cuidados relacionados ao dreno torácico. Portanto, a utilização de protocolos de assistência na utilização de drenos torácicos e práticas baseadas em evidências contribuem para a melhoria e qualidade da assistência de enfermagem a esses pacientes.

Descritores: Cuidados de Enfermagem; Drenagem; Parede Torácica; Sucção; Segurança do Paciente.

\section{Abstract}

The aim was to identify in the scientific literature the best nursing practices related to the use of a chest tube in an adult Intensive Care Unit. This is an integrative review with consultation of the Latin American Caribbean Literature on Health Sciences, Nursing Database, Online Medical Literature Search and Analysis System, Scientific Electronic Library Online, PubMed, CINAHL and EBSCO. Studies published between January 2014 and December 2018 in English, Portuguese and Spanish and available in full of full texts are included. Those with pediatric patients and duplicities were excluded. 133 studies were identified that gave rise to five articles analyzed. Of the selected articles, $80 \%$ corresponded to international studies; $40 \%$ published in 2016 and $60 \%$ with quantitative analysis. The categories of good practices with chest tube, complications related to chest tube, and use of a care protocol related to chest tube emerged. Therefore, the use of care protocols in the use of chest tubes and evidence-based practices contribute to the improvement and quality of nursing care for these patients.

Descriptors: Nursing Care; Drainage; Thoracic Wall; Suction; Patient Safety.

\section{Resumén}

El objetivo fue identificar en la literatura científica las mejores prácticas de enfermería relacionadas con el uso de un tubo torácico en una Unidad de Cuidados Intensivos de adultos. Se trata de una revisión integradora con consulta de la Literatura del Caribe Latinoamericano en Ciencias de la Salud, Base de Datos de Enfermería, Sistema de Búsqueda y Análisis de Literatura Médica en Línea, Biblioteca Electrónica Científica en Línea, PubMed, CINAHL y EBSCO. Se incluyen estudios publicados entre enero de 2014 y diciembre de 2018 en inglés, portugués y español y disponibles íntegramente con textos completos. Se excluyeron aquellos con pacientes pediátricos y duplicidades. Se identificaron 133 estudios que dieron lugar a cinco artículos analizados. De los artículos seleccionados, el $80 \%$ correspondió a estudios internacionales; $40 \%$ publicado en 2016 y 60\% con análisis cuantitativo. Surgieron las categorías de buenas prácticas con el tubo torácico, complicaciones relacionadas con el tubo torácico y uso de un protocolo de atención relacionado con el tubo torácico. Por lo tanto, el uso de protocolos de atención en el uso de sondas torácicas y prácticas basadas en la evidencia contribuyen a la mejora y calidad de la atención de enfermería para estos pacientes.

Descriptores: Cuidado de enfermería; Drenaje; Pared Torácica; Succión; Seguridad del Paciente. 
Boas práticas de enfermagem na utilização de dreno de tórax: revisão integrativa Hasselmann BNO, Ranção CS, Tavares GS, Almeida LF, Camerini FG, Paula VG

\section{Introdução}

Drenos torácicos correspondem a dispositivos invasivos utilizados para restabelecimento e manutenção da pressão negativa do espaço pleural, recuperando a função cardiopulmonar e estabilidade hemodinâmica. São utilizados com a finalidade de retirada de algum líquido sanguinolento, purulento, seroso ou para a remoção de $\operatorname{ar}^{1}$.

$\mathrm{Na}$ Unidade de Terapia Intensiva (UTI), a utilização de drenos torácicos refere-se à terapêutica empregada em casos de pós-operatórios de grandes cirurgias, traumas de tórax de origens variadas, tais como, derrame pleural, empiema, hemotórax, pneumotórax, sangramento, falha mecânica, edema. A colocação deste tipo de dispositivo tem como finalidade a retirada de fluidos, ar, sangue e secreção, por isso os cuidados com os drenos de tórax são de extrema relevância².

O uso de dreno de tórax pode levar a complicações, tais como, infecção, desposicionamento do dreno, retirada acidental, obstrução, sangramento, exteriorização entre outros, que podem gerar um aumento no tempo de hospitalização, morbidade, mortalidade. Um estudo apresenta que as principais complicações relacionadas ao uso do dreno de tórax são obstrução do sistema (58\%), seguido de enfisema (6\%), infecção peri-dreno, deslocamento acidental do dreno e pneumotórax $(1 \%)^{3}$.

Um outro estudo apontou que o paciente crítico em uso do dreno torácico pode apresentar outras complicações como o mau posicionamento do dreno e com necessidade de nova drenagem; hemotórax residual; pneumotórax residual; pneumonia; infecção e permanência do orifício fora da cavidade torácica ${ }^{4}$.

Por estarem hemodinamicamente instáveis, com tempo de internação prolongado e estarem expostos a procedimentos invasivos, os pacientes de UTI apresentam maior possibilidade de desenvolverem complicações e de maior severidade associadas ao uso do dreno de tórax ${ }^{5}$.

Diante dos problemas advindos do uso de dreno torácico e da necessidade de atualizar a assistência de enfermagem frente a manipulação deste dispositivo, este estudo teve como objeto as boas práticas no cuidado de enfermagem a pacientes críticos adultos que utilizam dreno torácico. Para tanto, traçou-se como objetivo identificar na literatura científica as melhores práticas de enfermagem relacionadas a utilização de dreno torácico em UTI adulta.

\section{Metodologia}

Estudo do tipo revisão integrativa, que possui como base a procura, avaliação crítica e a síntese das evidências sobre o tema de interesse, utilizando-se como método a investigação. Esse método fornece informações amplas sobre o assunto/problema, levando a formação de um abrangente corpo de conhecimento ${ }^{6}$. Na busca, foram identificados 133 artigos, que após a leitura na íntegra e critérios de inclusão, deram origem a cinco artigos, conforme Figura 1.

Figura 1. Fluxograma do processo de busca, seleção e análise dos artigos de revisão, adaptado do PRISMA. Rio de Janeiro, RJ, Brasil, 2021

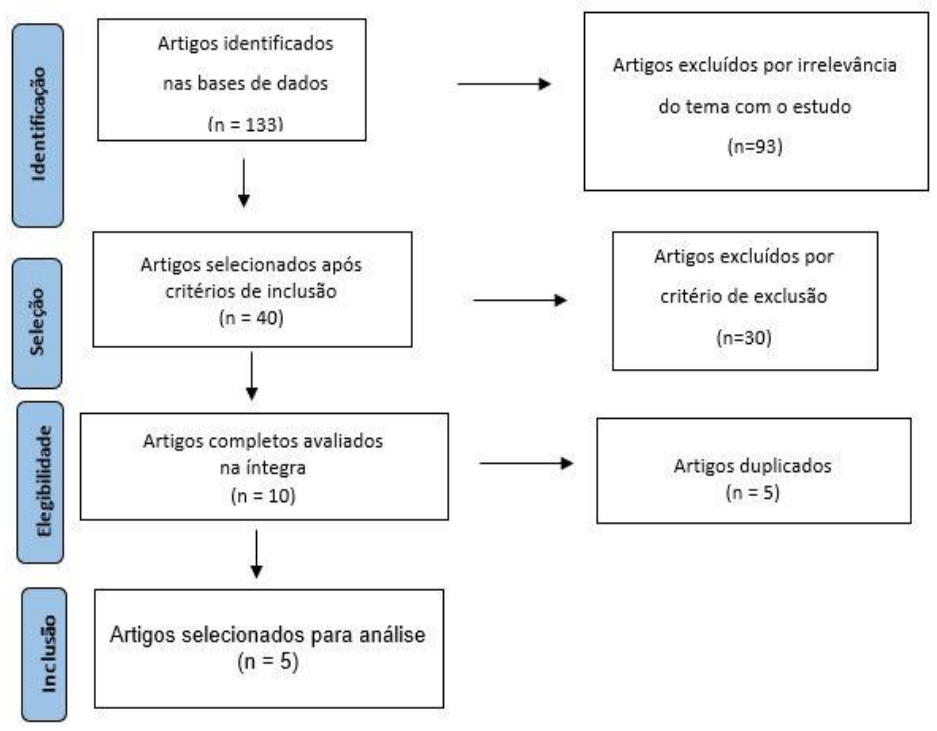

A revisão integrativa é constituída de seis fases que são a identificação do tema e seleção da hipótese ou questão de pesquisa, estabelecimento de critérios para inclusão e exclusão de estudos e a amostragem ou pesquisa na literatura, definição das informações a serem extraídas dos estudos selecionados e a categorização dos estudos; avaliação dos estudos incluídos; interpretação dos resultados; e apresentação da revisão/ síntese do conhecimento ${ }^{6}$.

Seguiu-se a estratégia PICo que tem como objetivo responder um problema com base em estudos de grande

parte quantitativos e que buscam a sensibilidade dos estudos qualitativos com foco no problema indicado por este estudo. A partir de quatro itens: a população, o paciente ou o problema abordado; a intervenção e a exposição que será considerada; a comparação da intervenção ou da exposição quando está for necessária e relevante; e os desfechos ou resultados clínicos de interesse ${ }^{7}$.

No caso deste estudo foram utilizados $\mathrm{P} / \mathrm{I} / \mathrm{Co}$, ou seja, $\mathrm{P}=$ pacientes na UTI adulta; $\mathrm{I}=$ melhores práticas de enfermagem e $\mathrm{Co}=$ dreno de tórax, se aplica devido ser uma revisão integrativa e ter a função do contexto de revisão? 
Assim, a questão que norteou a pesquisa foi quais as melhores práticas de enfermagem a pacientes com dreno de tórax na UTI adulta presentes na literatura?

Para tanto foi realizado um levantamento nos meses de abril a maio de 2019 nas bases de dados: Literatura Latino-Americana e do Caribe em Ciências da Saúde (LILACS), Base de Dados em Enfermagem (BDENF), Medical Literature Analysis and Retrievel System Online (MedLine), Scientific Electronic Library Online (SciELO), PubMed, Cumulative Index to Nursing and Allied Health Literature (CINAHL), Business Source Complete (EBSCO). Utilizados os Descritores em Ciências da Saúde (DECs) e Medical Subject Heading (MeSH): "Cuidados de Enfermagem", "Drenagem", "Tórax" e "Tubos Torácicos". Foi aplicado o operador booleano AND entre todos os descritores, a fim de se obter o cruzamento mais preciso na busca.

Foram incluídos estudos publicados no período de janeiro de 2014 a dezembro de 2018, nos idiomas inglês, português e espanhol, disponíveis na íntegra e textos completos. Excluídos aqueles relacionados a pacientes neonatais e pediátricos, e com duplicidades nas bases de dados.

Dos artigos identificados, procedeu-se à leitura minuciosa de cada estudo, destacando aqueles que
Boas práticas de enfermagem na utilização de dreno de tórax: revisão integrativa Hasselmann BNO, Ranção CS, Tavares GS, Almeida LF, Camerini FG, Paula VG responderam ao objetivo proposto por esta pesquisa, a fim de organizar e tabular os dados. Para tanto, foi elaborado instrumento de coleta de dados contendo base de dados, ano de publicação, periódico, país e idioma, tipo de estudo e nível de evidência, autores, título e cuidados de enfermagem a pacientes com dreno de tórax na UTI.

Procedeu-se, então, à caracterização dos estudos selecionados, sendo extraídos os conceitos abordados em cada artigo, conforme interesse. Os conteúdos foram comparados e agrupados por similaridade em categorias.

\section{Resultados}

Observou-se que dos cinco estudos, um é nacional (20\%) e quatro são internacionais (80\%). Em relação ao ano de publicação, foi evidenciado que dois estudos foram publicados no ano de 2016 (40\%); e um (20\%) em 2015, 2017 e 2018, respectivamente. Sobre o tipo de estudo foram encontrados, um estudo quanti-qualitativo, três quantitativo e um qualitativo, conforme Quadro 1. Após a leitura atenta do conteúdo dos artigos, emergiram três categorias: a) Boas práticas com dreno torácico, b) Complicações relacionadas ao dreno torácico e c) Uso de protocolo de cuidados relacionados ao dreno torácico.

Quadro 1. Categorização dos artigos selecionados segundo título, autor, periódico, tipo de estudo e cuidados de enfermagem ao paciente com dreno de tórax na UTI. Rio de Janeiro, RJ, Brasil, 2021

\begin{tabular}{|c|c|c|c|c|}
\hline № & Título/ Autor/Ano & Periódico & Tipo de estudo & $\begin{array}{l}\text { Boas práticas de enfermagem ao paciente com dreno e } \\
\text { tórax }\end{array}$ \\
\hline 1 & $\begin{array}{l}\text { Intervenção de Enfermagem: } \\
\text { cuidados com dreno torácico } \\
\text { em adultos no pós-operatório } \\
\text { (ALMEIDA et al, 2018) }\end{array}$ & Revista Rene & Quanti-qualitativo & $\begin{array}{l}\text {-Orientar o paciente nos cuidados com o dreno; } \\
\text { - Realizar troca do curativo a cada } 24 \text { horas; } \\
\text {-Manter o frasco de drenagem abaixo do nível do tórax; } \\
\text {-Realizar correta higienização durante manipulação do } \\
\text { dreno; } \\
\text {-Realizar ordenha do dreno quando necessário. }\end{array}$ \\
\hline 2 & $\begin{array}{l}\text { Place Atrium to Water Seal } \\
\text { (PAWS): Assessing Wall } \\
\text { Suction Versus No Suction } \\
\text { for Chest Tubes After Open } \\
\text { Heart Surgery } \\
\text { (KRUSE et al, 2017) } \\
\end{array}$ & Critical Care Nurse & Quantitativo & - Avaliar vazamento de ar e sinais de pneumotórax. \\
\hline 3 & $\begin{array}{l}\text { Institutional protocol to } \\
\text { standardize the chest drainage } \\
\text { system management, from } \\
\text { surgery to nursing care, at a } \\
\text { regional hospital in } \\
\text { northern Paraná. } \\
\text { (MORAIS et al, 2016) }\end{array}$ & $\begin{array}{l}\text { Acta Scientiarum. } \\
\text { Health Sciences }\end{array}$ & Quantitativo & $\begin{array}{l}\text {-Avaliar oscilação do selo d'água; } \\
\text {-Manter o frasco coletor no posicionamento correto; } \\
\text {-Realizar a ordenha. }\end{array}$ \\
\hline 4 & $\begin{array}{l}\text { Nurses' knowledge of care of } \\
\text { chest drain: A survey in a } \\
\text { Nigerian } \\
\text { semiurban university } \\
\text { hospital } \\
\text { (KESIEME et al, 2016) }\end{array}$ & $\begin{array}{l}\text { Annals of African } \\
\text { Medicine }\end{array}$ & Quantitativo & $\begin{array}{l}\text { - Manter sistema de drenagem abaixo do nível do tórax; } \\
\text { - Avaliar diariamente coloração do sistema de drenagem; } \\
\text {-Trocar o curativo sempre que necessário; } \\
\text {-Avaliar flutuação do sistema de drenagem; } \\
\text {-Realizar a ordenha do dreno. }\end{array}$ \\
\hline 5 & $\begin{array}{l}\text { Evidence-based management } \\
\text { of patients with chest tube } \\
\text { drainage system to reduce } \\
\text { complications in cardiothoracic } \\
\text { vascular surgery wards. } \\
\text { (GAN e TAN, 2015) }\end{array}$ & $\begin{array}{l}\text { International } \\
\text { Journal of Evidence- } \\
\text { Based Healthcare }\end{array}$ & Qualitativo & $\begin{array}{l}\text { - Avaliar local de inserção do dreno, atentando para } \\
\text { sinais flogísticos; } \\
\text {-Monitorar oscilação do frasco de drenagem } \\
\text { - Avaliar coloração do sistema de drenagem; } \\
\text { - Avaliar infiltração de ar; } \\
\text { - Manter curativo limpo e seco. }\end{array}$ \\
\hline
\end{tabular}




\section{Discussão}

\section{Boas práticas com dreno torácico}

A importância da correta manipulação do dreno caracteriza-se por intervenções de enfermagem como manter o frasco de drenagem abaixo do nível do tórax, clampear os drenos quando estiverem acima do nível do tórax e mantê-los dessa maneira no menor tempo possível, manter o sistema de drenagem no nível vertical, higienizar as mãos antes e após inserção do dreno, realizar troca do curativo a cada 24 horas ou quando necessário, monitorar sinais e sintomas de pneumotórax, realizar a limpeza ao redor do dreno com solução fisiológica a 0,9\%, utilizar gaze com soro, secar e ao redor realizar a limpeza com álcool a $70 \%$, utilizar clampes não traumáticos, monitorar através da radiografia o posicionamento do dreno, tempo de troca dos frascos de drenagem torácica e trocar os frascos de sistema de drenagem sempre que necessário; realizar ordenha quando indicado orientar o paciente e a família sobre a necessidade de cuidados com o dreno; evitar a oclusão do frasco. Em relação à ordenha e à utilização de clampes ainda há uma escassez de pesquisas, demonstrando se pode ou não ser realizada².

Ainda a ordenha da tubulação do dreno de tórax deve ser sempre realizada no sentido da incisão para o frasco coletor, na presença de coágulo e com frequência de $2 / 2 \mathrm{~h}-$ $4 \mathrm{~h} / 4 \mathrm{~h}$ ou de acordo com o protocolo da instituição ${ }^{8}$.

Um estudo evidência uma baixa adesão relacionada a algumas práticas com o dreno, tais como, ordenha, utilização de clampes não traumáticos, tempo de troca dos frascos de drenagem torácica, orientação a família e ao paciente. Em relação à ordenha e a utilização de clampes, ainda há uma escassez de pesquisas, demonstrando se pode ou não ser realizada. E ainda há dúvidas sobre a troca do sistema de drenagem².

Em relação às trocas do sistema de drenagem, o profissional deverá informar ao paciente e a família sobre o procedimento, além de fazer antissepsia das mãos, separar todo o material a ser utilizado, abrir os frascos de solução salina ou água destilada, fazer novamente antissepsia das mãos e calçar luvas, encher o frasco coletor com solução até cobrir $2 \mathrm{~cm}$ do tubo rígido, marcar o nível do líquido com adesivo e anotar data e hora da troca do selo d'água, podendo usar uma pinça hemostática para pinçar o dreno antes da troca do circuito ${ }^{8,9}$.

Durante o transporte do paciente um cuidado especial deve ser dispensado ao frasco coletor, devendo sempre abaixo do nível do tórax do paciente para evitar que coleções retornem à cavidade pleural do paciente. Outro fator importante diz respeito ao lacre do tubo de extensão, que não deve ser fechado durante o transporte ${ }^{9}$.

Em relação a isso, pacientes com pneumotórax com grande escape aéreo, rapidamente podem evoluir para pneumotórax hipertensivo se o lacre do sistema for fechado. Assim, o dreno só deve ser fechado por frações de segundos em situações que o frasco coletor fique acima do nível do tórax, e logo em seguida ser aberto. Além disso, segundo os autores, não há evidência científica que a ordenha do sistema possa contribuir para prevenção da obstrução do sistema ${ }^{8}$.
Um estudo analisou o conhecimento que os enfermeiros possuem sobre o cuidado com dreno de tórax, em um hospital da Nigéria. Para tanto, foram utilizadas variáveis como sexo, idade, setor, qualificação acadêmica, tempo de profissão, e se os profissionais receberam treinamento ou participaram de evento com essa temática. A maior parte dos profissionais (99\%) sabia corretamente o local de inserção e a finalidade do uso do dispositivo, demonstrando conhecimento básico de anatomia e da função do dreno torácico. Os autores verificaram a necessidade de treinamento em relação a essa prática, principalmente no manejo pós-operatório, onde foi evidenciado menor habilidade ${ }^{10}$.

Embora os profissionais de enfermagem acreditem na importância de treinamento sobre dreno torácico, a maior parte $(66,7 \%)$ não recebeu treinamento para se atualizar no assunto, sendo um fator preocupante, visto que a manutenção do dreno torácico é atribuição do enfermeiro. Além disso, cerca de $45 \%$ dos enfermeiros não sabiam ou não estavam certos de que a flutuação do nível de fluido na tubulação de drenagem era um indicativo de bom funcionamento do dispositivo. A grande maioria reconheceu que o sistema de drenagem deve estar abaixo do nível da inserção do dreno. Outro cuidado abordado foi com relação à ordenha, para se evitar a obstrução do sistema de drenagem. Foi observado que $41,1 \%$ dos sujeitos acreditavam que a ordenha deve ser realizada pelo enfermeiro ${ }^{10}$.

Pode-se perceber a importância dos estudos na elaboração de estratégias a fim de melhorar a assistência no cuidado com dreno de tórax. Duas pesquisas evidenciaram falhas na correta manipulação e manejo com esse dispositivo, demonstrando a importância da pesquisa relacionada a essa prática, uma vez que, a equipe de enfermagem tem grande responsabilidade no manuseio dos drenos torácicos ${ }^{2,11}$.

Tendo em vista que o manejo e manutenção do dreno é uma prática realizada pela equipe de enfermagem, é de suma importância a discussão sobre essa temática. Contudo há uma escassez de pesquisas relacionadas com os cuidados na manutenção do dreno torácico e prevenção de complicações.

Sobre os cuidados de enfermagem, os pacientes submetidos a drenagem pleural de preferência são internados nas enfermarias onde realizam troca de curativos, aferição dos débitos do dreno, troca de selo d'água e acompanhamento de sua evolução. Entretanto em outros setores como politrauma e observação cirúrgica são setores que há grande número de pessoas circulantes, o que pode propiciar um aumento de contaminações e possíveis infecções desses pacientes ${ }^{12}$.

A troca de curativo pelos enfermeiros é importante, uma vez que estes devem avaliar o sítio de inserção e sinais flogísticos, a fim de evitar possíveis complicações, como infecção $o^{2,11}$. Alto índice de validação dos enfermeiros em relação a essa prática foi evidenciado. No entanto, há um alto índice de utilização de apenas álcool a 70\% para realização do curativos ${ }^{2}$. Contudo, pode-se utilizar solução fisiológica a $0,9 \%{ }^{13}$. 
O enfermeiro deve encorajar a mobilização do paciente sempre que possível para facilitar a drenagem, realizar a ordenha do dreno na direção do frasco de drenagem evitando que ele se obstrua como coágulos e/ou fibrina, promover a respiração profunda e a tosse em intervalos frequentes permitindo eliminação dos fluidos acumulados e a expansão pulmonar, evitando a atelectasia $^{14}$.

Assim, como resultado, uma lista de verificação com o intuito de ajudar na melhoria da prática relacionada com o dreno de tórax, pode ser utilizada. Essa lista foi aplicada por enfermeiros e houve uma melhoria na assistência, pois estes eram alertados em relação a possíveis complicações, através de um checklist realizado diariamente. Vale ressaltar as dificuldades de adesão pelos enfermeiros referente a lista de verificação, pois alegaram excesso de trabalho havendo dificuldade em aceitar a mudança ${ }^{11}$.

\section{Complicações relacionadas ao dreno torácico}

A gestão de drenos torácicos varia e afeta o tempo de permanência dos drenos, o manejo da dor, tempo de internação, a mobilização precoce dos pacientes, e o tipo de cuidados de enfermagem que são necessários ${ }^{15}$.

Os autores trazem à tona os pacientes adultos que realizam cirurgias cardíacas e que utilizam tubos torácicos para drenar o excesso de líquidos, ar e sangue, ou criar sucção por pressão negativa adicional para reexpansão pulmonar. Nessas cirurgias, a colocação de pelo menos um tubo de mediastino é tradicional, porém os pacientes também podem ter um segundo tubo torácico pleural ${ }^{15}$.

O preenchimento de critérios para avaliação de drenos torácicos com selo de água mostra-se importante até que o tubo torácico seja removido. Os cuidados de enfermagem devem ser direcionados a possíveis vazamentos de ar pelos drenos torácicos, dispneia aguda, aumento de drenagem ao redor do peito local de inserção do tubo, alterações do ritmo - atrial fibrilação e taquicardia ${ }^{15}$.

Quanto à localização, pode-se dizer que os tubos torácicos no mediastino são colocados sobre a superfície do diafragma no pericárdico, enquanto os tubos torácicos pleurais são colocados no espaço pleural, sendo pressão negativa no espaço pleural e o sistema fechado com pressões intrapleural de aproximadamente $4 \mathrm{mmHg}$ entre respirações. Portanto, essa pressão negativa permite a expansão e mantém no pulmão durante a inspiração, no entanto a entrada de ar, fluido, ou do sangue para dentro do espaço interrompe a pressão negativa, e o pulmão recua-se e colapsa. Contudo, pacientes submetidos a outros tipos de cirurgia torácica como ressecção pulmonar, também podem ter tubos torácicos pleurais colocado no pós-operatório para prevenir o pneumotórax, para monitorizar vazamento de ar e o hemotórax ${ }^{15}$.

Historicamente, a técnica de drenagem torácica começou a ser utilizada através da inserção de um tubo metálico no espaço pleural acoplado a um frasco coletor. No entanto, atualmente corresponde à inserção de um tubo de cloreto de polivinil ou de silicone no qual uma de suas extremidades é inserida na cavidade pleural, outra no frasco
Boas práticas de enfermagem na utilização de dreno de tórax: revisão integrativa Hasselmann BNO, Ranção CS, Tavares GS, Almeida LF, Camerini FG, Paula VG ou equipamento coletor dando origem ao mecanismo valvular unidirecional. Esse mecanismo faz com que o conteúdo drenado da cavidade pleural não retorne para a cavidade pleural, assim restabelecendo a recuperação da expansão e da função pulmonar nas condições fisiológicas da cavidade pleural na medida em que restabelece a pressão negativa dentro do tórax ${ }^{14}$.

Desse modo, a equipe de enfermagem possui grande importância na manutenção dos drenos torácicos, assegurando que a aspiração esteja funcionando e os tubos estejam permeáveis, realizando a vigilância do sistema para que não corra fuga de ar do sistema, através de possíveis borbulhamentos e garantido que haja flutuação do nível do líquido no tubo de drenagem ${ }^{14}$.

Portanto, a verificação do débito drenado é crucial, pois avalia o quanto de sangue ou líquido o paciente está perdendo após o trauma. Pode-se ser feito a cada hora ou de 2/2 horas para avaliar pacientes graves, ressaltando que $1500 \mathrm{ml}$ de sangue na drenagem inicial é indicação de toracotomia de emergência, bem como débitos de 200 $\mathrm{ml} /$ hora por 2 a 4 horas $^{16}$.

Pacientes graves devem ser submetidos à aferição do débito de hora em hora e ter seus sinais vitais avaliados de modo contínuo. Pacientes estáveis na enfermaria ou em UTI podem ter seu débito aferido a cada 24 horas $^{9}$.

Dentre as complicações relacionados ao uso do dreno torácico estão: mau posicionamento do dreno e com necessidade de nova drenagem; hemotórax residual; pneumotórax residual; pneumonia; infecção e permanência do orifício fora da cavidade torácica ${ }^{4}$.

Em um estudo, a obstrução do sistema de drenagem ocorreu em 6\% (6/100) dos casos, causada por clampeamento (um caso), sifonagem (dois casos) e coágulos e fibrina (três casos). Não houve associação estatística significativa entre um período de drenagem mais prolongado com a maior ocorrência de mecanismos de obstrução relacionados. Em relação à drenagem torácica, foi $5 \%$ (5/100) de enfisema subcutâneo - devido ao dreno localizado no tecido celular subcutâneo; um caso (1\%) de infecção ao redor o dreno; cinco casos (5\%) de deslocamento acidental do tubo, necessitando reidratação, e em 5\% (5/100) dos pacientes houve relatos ao remover o dreno, com pneumotórax devido à presença de ar na cavidade pleural no momento da remoção; no entanto a redrenagem não foi necessária ${ }^{17}$.

Em uma outra pesquisa também foi possível observar como complicações: obstrução do sistema (58\%), enfisema (6\%), infecção peri-dreno, deslocamento acidental do dreno e pneumotórax $(1 \%)^{3}$.

Os pacientes de UTI apresentam maior possibilidade de desenvolverem complicações e de maior severidade associadas ao uso do dreno de tórax, por estarem hemodinamicamente instáveis, com tempo de internação prolongado e estarem expostos a procedimentos invasivos 5 .

\section{Uso de protocolo de cuidados relacionados ao dreno torácico}

Em relação ao manejo dos sistemas de drenagem, a padronização e implementação de um protocolo para os 
Boas práticas de enfermagem na utilização de dreno de tórax: revisão integrativa Hasselmann BNO, Ranção CS, Tavares GS, Almeida LF, Camerini FG, Paula VG os critérios de inserção do dreno, conexão do tubo, borbulhamento, mudança de coloração da água, pressão de sucção.

Vale ressaltar as dificuldades de adesão pelos enfermeiros referente à lista de verificação, pois alegaram excesso de trabalho havendo dificuldade em aceitar a mudança. Em suma, foi observado que o uso de um guia para os enfermeiros gera mais segurança durante o manuseio com os drenos torácicos ${ }^{11}$.

Os artigos analisados vão de encontro com o objetivo dessa pesquisa, como as boas práticas no cuidado de enfermagem a pacientes críticos adultos com dreno torácico, demonstrando a importância do conhecimento dos enfermeiros sobre os cuidados com o dispositivo e a sugestão da utilização de um protocolo pré-estabelecido.

Assim, diante do conteúdo descrito foi elaborado um checklist, contendo os seguintes itens a serem observados pela equipe de enfermagem no cotidiano da UTI quando há pacientes, neste setor, em uso de drenos torácicos. São eles: curativo, drenagem, cuidados gerais e a resposta do paciente ao uso do dreno.

Quanto ao curativo, o instrumento permite avaliar se ele foi realizado nas últimas 24 horas, se está limpo e seco, se o óstio apresenta ou não sem sinais flogísticos, se há fixação/sutura adequada. Em relação à na drenagem, é possível registrar a permeabilidade, o aspecto, o volume, presença de coágulo, oscilação e borbulha.

Outros cuidados gerais compreendem, confirmar se o dreno está livre de tração ou pinçamento, data e hora da troca da água destilada no frasco coletor, marcação da altura da água destilada $(2 \mathrm{~cm})$ no selo d'água, posição do frasco coletor em relação ao piso/fixado na cama ou suporte, bem como nível do tórax. Em relação à resposta do paciente ao uso do dreno, o instrumento permite confirmar orientação quanto ao uso, presença de dor e o padrão respiratório. Tal instrumento permite sistematizar a assistência de enfermagem nos cuidados relativos a pacientes críticos que utilizam o dreno torácico. Ademais, para além do uso adequado do instrumento, o registro em prontuário pode indicar se a assistência de enfermagem foi prestada de forma segura ao paciente ${ }^{18}$.

\section{Conclusão} pacientes com sistema de drenagem torácica em enfermarias cardiotorácicas em um hospital universitário de Singapura, um estudo mostrou que ocorreram dez incidentes sobre desposicionamento do dreno e cinco relacionados à fixação do dreno ${ }^{11}$.

Diante disso, foi elaborada uma lista de verificação, com o intuito de ajudar na melhoria da prática relacionada com o dreno de tórax. Essa lista foi aplicada a enfermeiros a fim de auxiliá-los na manipulação do dreno, desde a sua inserção até o final da drenagem. No primeiro momento, foram evidenciadas 36 falhas relacionadas à sucção, borbulhamento e mudança de cor. Após a lista de verificação, houve uma melhoria na assistência, pois os enfermeiros eram alertados em relação a possíveis complicações, através de um checklist realizado diariamente ${ }^{11}$. A verificação do dreno torácico pode seguir

O estudo atingiu o objetivo proposto, identificando
na literatura as melhores evidências relacionadas as boas práticas de enfermagem na utilização de dreno torácico na UTI adulta. Frente às possíveis complicações do uso do dreno de tórax no paciente crítico, que interferem negativamente no seu processo saúde-doença, este estudo possibilita a assistência de enfermagem mais direcionada a esses pacientes, uma vez que, promove orientações embasadas acerca do cuidado com o dreno de tórax.

Como limitações verificou-se um número pequeno de artigos encontrados nas bases de dados. Diante disso, a produção de mais estudos científicos com essa temática pelos profissionais de enfermagem, com a finalidade de garantir uma assistência de qualidade e segura é necessária. 
Conforme visto nessa revisão de literatura, dois estudos apontam para utilização de protocolos de assistência na utilização de drenos torácicos e todos os outros confirmam que práticas baseadas em evidências
Boas práticas de enfermagem na utilização de dreno de tórax: revisão integrativa Hasselmann BNO, Ranção CS, Tavares GS, Almeida LF, Camerini FG, Paula VG contribuem para a melhoria e qualidade da assistência de enfermagem a esses pacientes submetidos a drenagem por drenos torácicos.

\section{Referências}

1. Carvalho EA, Oliveira MVB. Modelo de segurança para realização de drenagem torácica na pandemia pela COVID-19. Rev. Col. Bras. Cir. 2020;47:e20202568. DOI: 10.1590/0100-6991e-20202568

2. Almeida RC, Souza PA, Santana RF, Luan AA. Intervenção de enfermagem: cuidados com dreno torácico em adultos no pós-operatório. Rev Rene. 2018;19:e3332. DOI: 10.15253/2175-6783.2018193332

3. Nishida G, Sarrão BD, Colferai DR, Tenório GOS, Bandeira COP. Cuidados com o sistema de drenagem torácica em adultos internados no Hospital Universitário Regional de Maringá, Estado do Paraná, Brasil. Acta Sci. Health Sci. 2011;33(2):173-9. DOI: 10.4025/actascihealthsci.v33i2.8877

4. Mendes CA, Hirano ES. Fatores preditores de complicações da drenagem de tórax em pacientes vítimas de trauma. Rev. Col. Bras. Cir. 2018;45(2):e1543. DOI: 10.1590/0100-6991e-20181543

5. Roque KE, Tonini T, Melo ECP. Eventos adversos na unidade de terapia intensiva: impacto na mortalidade e no tempo de internação em um estudo prospectivo. Cad. Saúde Pública. 2016;32(10). DOI: 10.1590/0102-311X00081815

6. Sousa LMM, Vieira CMAM, Severino SSP, Antunes AVA. Metodologia de revisão integrativa da literatura em enfermagem. Revista investigativa em enfermagem [Internet]. 2017 [acesso em 05 set 2020];(2). Disponível em: http://hdl.handle.net/20.500.12253/1311

7. Oliveira AWC. Recuperação da informação em saúde: construção, modelos e estratégias. ConCl. [Internet] 2020 [acesso em 05 set 2020];3(2). Disponível em: https://seer.ufs.br/index.php/conci/article/view/13447

8. Kusahara DM, Chanes DC, Harada MJCS. Boas Práticas - Drenos de Tórax. Conselho Regional de Enfermagem de São Paulo [Internet]. 2011[acesso em 05 set 2020]. Disponível em: https://portal.coren-sp.gov.br/sites/default/files/dreno-de-torax.pdf

9. Medeiros BJC. Cuidados Padronizados com Dreno de Tórax: Aspectos e Manejo [Dissertação]. Profissional em Cirurgia Universidade Federal do Amazonas [Internet]. Amazonas; 2019 [acesso em 27 set 2020]. Disponível em: https://tede.ufam.edu.br/handle/tede/7165

10. Kesieme EB, Essu IS, Arekhandia BJ, Welcker K, Prisadov G. Nurses' knowledge of care of chest drain: A survey in a Nigerian semiurban university hospital. Ann Afr Med. 2016;15(2):95. DOI: 10.4103/1596-3519.172556

11. Gan KL, Tan M. Evidence-based management of patients with chest tube drainage system to reduce complications in cardiothoracic vascular surgery wards. Int J Evid Based Healthc. 2015;13(2):58-65. DOI: 10.1097/XEB.0000000000000041

12. Conselho Regional de Enfermagem de São Paulo (COREN - SP). Parecer no. 035/2019. Retirada de drenos de diferentes tipos, troca de selo d'água, ordenha e aspiração contínua por profissional de enfermagem. [Internet]. São Paulo (SP): COREN; 2019 [acesso em 20 set 2020]. Disponível em: https://portal.coren-sp.gov.br/wp-content/uploads/2020/01/Parecer-035.2019-Manuseio-de-drenos-pelaenfermagem-retirada-troca-de-selo-d\%C3\%A1gua-ordenha-e-aspira\%C3\%A7\%C3\%A3o.pdf

13. Brasil. Agência Nacional de Vigilância Sanitária. Medidas de Prevenção de Infecção Relacionada à Assistência à Saúde [Internet]. Brasília (DF): Anvisa;2017 [acesso em 20 set 2020]. Disponível em: https://www20.anvisa.gov.br/segurancadopaciente/index.php/publicacoes/item/caderno-5

14. Lucio V, Araújo APS. Nursing care in thoracic drainage: a literature review. UNOPAR. Cient., Ciênc. biol. Saude [Internet]. 2011;[acesso em 20 set 2020]13. Disponível em: https://edisciplinas.usp.br/pluginfile.php/5585868/mod_resource/content/1/Lucio\%20e\%20Araujo\%202011.pdf

15. Kruse T, Wahl S, Guthrie PF, Sendelbach S. KRUSE, T. et al. Place Atrium to Water Seal (PAWS): Assessing Wall Suction Versus No Suction for Chest Tubes After Open Heart Surgery. Critical Care Nurs. 2017;37(4):17-28. DOI: 10.4037/ccn2017269

16. American College of Surgeons (ACS). ATLS Advanced Trauma Life Support: Student course manual [Internet]. 2018 [acesso em 20 set 2020]. Disponível em: http://paginas.facmed.unam.mx/deptos/cirugia_/wp-content/uploads/2018/07/Advanced-Trauma-LifeSupport.pdf

17. Morais ACC, Lemos MM, Marques VD, Bandeira COP. Protocolo institucional para padronizar o manejo do sistema de drenagem torácica, da cirurgia ao cuidado de enfermagem, em um hospital regional do norte do Paraná. Acta Scientiarum. Health Sciences. 2016;38(2): 17377. DOI: https://doi.org/10.4025/actascihealthsci.v38i2.26972

18. Queiroz FSMA, Moraes SC, Nogueira VSAL, da Silva OT, Duarte de OL. Auditoria de enfermagem: aspectos da qualidade da assistência e do seu registro no prontuário. Glob Acad Nurs. 2020;1(2):e33. DOI: 10.5935/2675-5602.20200033 